\title{
Consumo de alcohol, tabaquismo y características sociodemográficas en estudiantes universitarios
}

\author{
Alcohol, tobacco consumption and sociodemographic features associated in college students
}

\author{
Sonia Carolina Mantilla-Toloza', Carmen Edilia Villamizar², Karl Peltzer $^{3}$
}

1 PhD. en Ejercicio físico, Fisioterapia y Salud. Docente Facultad de Salud, Universidad de Pamplona. Pamplona, Colombia. email: sonia.mantilla@unipamplona.edu.co

2 Mg. en Educación Matemática. Docente Facultad de Ciencias Básicas, Universidad de Pamplona. Pamplona, Colombia. email: cevill@unipamplona.edu.co

3 Investigador Departamento de Psicología. University of the Free State, Bloemfontein 9300, South Africa. Director HIV/AIDS/STIs and TB (HAST) Research Programme, Human Sciences Research Council, Private Bag X41, Pretoria 0001, South Africa. email karl.pel@mahidol.ac.th

Mantilla-Toloza SC, Villamizar CE, Peltzer K. Consumo de alcohol, tabaquismo y características sociodemográficas en estudiantes universitarios. Univ. Salud. 2016;18(1):7-15. DOI: http://dx.doi.org/10.22267/rus.161801.14

\section{Resumen}

Introducción: Los estudiantes universitarios son altamente vulnerables a la adopción de hábitos que representan riesgos para su salud, incluyendo el tabaquismo y consumo de alcohol que inciden en la ocurrencia de enfermedades crónicas; por esto se requiere de información preliminar que permita identificar los factores asociados a éstos hábitos para la planificación de estrategias de intervención desde el contexto universitario. Objetivo: Identificar asociaciones entre el consumo de alcohol, el tabaquismo y características demográficas en un grupo de universitarios. Materiales y métodos: Estudio descriptivo, transversal, con una muestra de 360 hombres y 456 mujeres (obtenida por muestreo aleatorio estratificado) a quienes se aplicó una encuesta para caracterizar el consumo de alcohol, tabaquismo y los factores sociodemográficos. La recolección de información se realizó bajo la supervisión y aprobación del Comité de Ética de la Universidad de Pamplona. El análisis de datos incluyó distribución de frecuencias en relación al tabaquismo, consumo de alcohol y otros factores y prueba $\mathrm{V}$ de Cramer para establecer asociación entre variables. Resultados: La prevalencia de tabaquismo es de $15 \%$ y de consumo frecuente de alcohol es de $26 \%$. Se encontraron asociaciones entre sexo y consumo de alcohol $(\mathrm{p}<0,001)$ y tabaquismo $(\mathrm{p}<0,001)$; siendo los hombres mayores consumidores. También se encontró asociación entre facultad y consumo de alcohol $(p=0,018)$ y tabaquismo $(p<0,001)$. Conclusiones: El consumo de tabaco y alcohol fue bajo en relación con estudios previos. Las variables sexo y facultad están asociadas al consumo de tabaco y alcohol. Se requiere implementar programas de optimización para la prevención y disminución del tabaquismo y consumo de alcohol en universitarios.

Palabras clave: Evaluación; hábito de fumar; bebidas alcohólicas; estilo de vida; diagnóstico; estudiantes universitarios. (Fuente: DeCS, Bireme).

\begin{abstract}
Introduction: College students are highly vulnerable to the adoption of habits that pose risks to their health, including smoking and alcohol consumption which have an impact on the occurrence of chronic diseases. Therefore, preliminary information that allows to identify the factors associated with these habits is required for the planning of intervention
\end{abstract}


strategies from the university context. Objective: To identify associations between alcohol consumption, smoking and demographic features in a group of college students. Materials and methods: A cross-sectional descriptive study was used with a sample of 360 men and 456 women (obtained by stratified random sampling). A survey was applied to them in order to establish alcohol consumption, tobacco use and demographic features. The data collection was carried out under the supervision and approval of the Ethics Committee of the University of Pamplona. The data analysis included frequency distribution in relation to smoking, alcohol consumption and other factors, and V Cramer test to establish association between variables. Results: The prevalence of tobacco is $15 \%$ and frequent alcohol consumption prevalence is $26 \%$. Association between gender and alcohol consumption $(\mathrm{p}<0,001)$ and tobacco use $(\mathrm{p}<0,001)$ was found, where men are the most consumers. Besides, association between faculty and alcohol consumption $(\mathrm{p}=0,018)$ and tobacco use $(p<0,001)$ was found. Conclusions: Tobacco and alcohol consumption was low in relation to prior studies. The variables of gender and faculty are associated to tobacco use and alcohol consumption. It is necessary to implement programs to prevent and decrease tobacco use and alcohol consumption in college students.

Keywords: Evaluation; smoking; alcoholic beverages; lifestyle; diagnosis; college students. (Source: DeCS, Bireme).

\section{Introducción}

Se ha reconocido que el consumo de tabaco está relacionado con la ocurrencia de enfermedades crónicas, las cuales representan la mayor carga de morbilidad y mortalidad a nivel mundial.1-3

A su vez, el consumo excesivo de alcohol ocasiona 3,3 millones de muertes en el mundo cada año. En el grupo etario de 20 a 39 años, un $25 \%$ de las defunciones son atribuibles al consumo de alcohol; además que es el causante de más de 200 enfermedades, provocando disfunción y discapacidad a una edad relativamente temprana.4

Por otra parte, se ha reconocido que los hábitos de vida empiezan a formarse en edades tempranas hasta constituirse como patrones de comportamiento en la vida adulta. Durante el período de transición, los adultos jóvenes, generalmente universitarios, son altamente vulnerables frente al impacto que ocasiona la influencia de factores determinantes de estilos de vida.5

Tradicionalmente, los jóvenes ingresan en la universidad cerca de los 18 años, enfrentándose a los cambios que llevan consigo la vida académica, los nuevos amigos y el ambiente que les rodea. Diversas investigaciones han demostrado que, en general, ellos adoptan hábitos que representan riesgos para su salud, incluyendo el tabaquismo, consumo de alcohol, inactividad física, prácticas alimentarias no saludables, entre otros.6-8
El presente trabajo se realizó con el objetivo de describir la prevalencia de consumo de tabaco y alcohol en universitarios y determinar asociaciones con características sociodemográficas como edad, sexo, año de estudio, facultad y desempeño académico; mediante las cuales se pretende identificar si podrían suponer situaciones de riesgo para la adopción y/o el mantenimiento de dichos hábitos, tal como se ha establecido en estudios previos, en donde el sexo masculino, el ingreso a la universidad, cursar los primeros años académicos de vida universitaria y tener un desempeño académico bajo, se relacionan con un mayor consumo de tabaco y alcohol en la universidad.5,6,9-13

Los resultados derivados de éste análisis constituirán una línea de base para el diseño, planificación e implementación de estrategias que promuevan la disminución y prevención del consumo de éstas sustancias desde el contexto universitario.

\section{Materiales y métodos}

El estudio descriptivo es de tipo transversal, en el cual se establecieron asociaciones con algunas variables sociodemográficas.

\section{Población y muestra}

La población estuvo conformada por 8.022 estudiantes matriculados en el segundo semestre académico del año 2012, pertenecientes a la sede 
central de la Universidad de Pamplona, de los cuales 4.145 eran hombres y 3.877 eran mujeres. Para determinar el tamaño de muestra se tuvo en cuenta la totalidad de estudiantes ya enunciada, un nivel de confianza del 95\% y error de estimación del 3,26\%.

Se aplicó muestreo aleatorio estratificado. Los tamaños de muestra en cada estrato se tomaron proporcionales al tamaño del mismo, estratificando la población por sexo y facultad. De lo anterior se obtuvo un tamaño de muestra mínima para hombres de 352 y para mujeres de 350. La muestra final estuvo conformada por 360 hombres y 456 mujeres, excluyendo aquellos universitarios menores de 18 años.

\section{Instrumento}

El instrumento empleado para la obtención de la información fue el "Questionnaire Health and Lifestyle" diseñado para el "Health behaviour survey among university students in low and middle income countries: a multi-country study". Este cuestionario fue elaborado a partir del "Global school health survey". 14

El cuestionario fue traducido, revisado por expertos y validado previa aplicación (coeficiente alfa de Cronbach entre 0,743 y 1,000). La información obtenida incluye datos sociodemográficos (edad, sexo, facultad, nivel económico familiar), desempeño académico, percepción del estado de salud, consumo de tabaco y de alcohol.

Se realizó estratificación de la edad, aplicando el método de la raíz cuadrada de la frecuencia relativa lo que permite construir grupos etareos más significativos que si se formaran según criterio del investigador.

Para ser categorizado como fumador, el estudiante debía indicar que actualmente es consumidor de uno o más productos derivados del tabaco, marcando la opción "si". En tal caso debía indicar la frecuencia de consumo de uno o más cigarrillos en el mes pasado: una o dos veces, semanalmente, casi diariamente o diariamente. Respecto al alcohol, el estudiante debía seleccionar si se describe como no bebedor, bebedor muy ocasional (solo ocasiones especiales), bebedor ocasional $\mathrm{o}$ bebedor frecuente.

Si se describió como bebedor ocasional o frecuente debía registrar cuántos días en las últimas dos semanas había bebido. Para facilitar el análisis se agruparon las cuatro dimensiones de consumo de alcohol sólo en dos: bebedor frecuente (incluye las opciones bebedor ocasional o frecuente) y no bebedor (no bebedor, bebedor muy ocasional).

"Health behaviour survey among university students in low and middle income countries: a multi-country study". Este cuestionario fue elaborado a partir del "Global school health survey". ${ }_{14}$

\section{Procedimiento y análisis de la información}

La recolección de la información se realizó entre los meses de octubre a diciembre de 2012, bajo la supervisión y aprobación del Comité de Ética e impacto ambiental de la Universidad de Pamplona. La recopilación de información se llevó a cabo durante horas de clase, con los estudiantes que estuvieran presentes, exceptuando menores de edad, o a quienes decidieran no participar o ya hubieran contestado la encuesta en otro grupo.

Previa autorización por el docente responsable de la clase respectiva, los estudiantes diligenciaron el consentimiento informado y la encuesta diseñada para el estudio. Se aseguró a los participantes anonimato y confidencialidad. Seguidamente los datos obtenidos fueron consignados en un formato de ingreso.

El análisis estadístico incluyó distribución de frecuencias en relación al tabaquismo, consumo de alcohol, sexo y otros factores; así como también la prueba V de Cramer para establecer asociación entre el sexo vs consumo de tabaco, alcohol y demás variables; teniendo en cuenta que están medidas en escala nominal y que la mayoría de ellas tienen más de dos categorías. 


\section{Resultados}

La edad promedio de los estudiantes que participaron en el estudio fue de 21 años (DT: $3,07)$. La mayoría de estudiantes pertenecen al estrato medio-bajo y bajo, mientras que la mitad considera que su desempeño académico y estado de salud es bueno. La tabla 1 muestra las características de los sujetos, a partir del sexo (Tabla 1).

Tabla 1. Características sociodemográficas por sexo estudiantes universitarios. Universidad de Pamplona - 2012

\begin{tabular}{|c|c|c|c|}
\hline Característica & $\begin{array}{c}\text { Total } \\
\mathrm{n}=\mathbf{8 1 6}(\mathbf{1 0 0 \% )})\end{array}$ & $\begin{array}{c}\text { Hombres } \\
n=360(44 \%)\end{array}$ & $\begin{array}{c}\text { Mujeres } \\
\mathrm{n}=456(56 \%)\end{array}$ \\
\hline $\begin{array}{l}\text { Nivel socioecond } \\
\text { Alto }\end{array}$ & $1(0,1)$ & $1(0,1)$ & $0(0)$ \\
\hline Medio & $103(12,6)$ & $48(5,9)$ & $55(6,7)$ \\
\hline Medio bajo & $498(61)$ & 220 (27) & $278(34,1)$ \\
\hline Bajo & $214(26,2)$ & $91(11,2)$ & $123(15,1)$ \\
\hline \multicolumn{4}{|l|}{ Facultad } \\
\hline Artes & $81(9,9)$ & $35(4,3)$ & $46(5,6)$ \\
\hline Agrarias & $25(3,1)$ & $20(2,5)$ & $5(0,6)$ \\
\hline Básicas & $36(4,4)$ & $17(2,1)$ & $19(2,3)$ \\
\hline Económicas & $43(5,3)$ & $15(1,8)$ & $28(3,4)$ \\
\hline Educación & $17(2,1)$ & $49(6)$ & $66(8,1)$ \\
\hline Ingenierías & $251(30,8)$ & $157(19,2)$ & $94(11,5)$ \\
\hline Salud & $314(38,5)$ & $99(12,1)$ & $215(26,3)$ \\
\hline \multicolumn{4}{|l|}{ Año de estudio } \\
\hline 1 & $204(25)$ & $97(11,9)$ & $107(13,1)$ \\
\hline 2 & $233(28,6)$ & $91(11,2)$ & $142(17,4)$ \\
\hline 3 & $165(20,2)$ & $66(8,1)$ & $99(12,1)$ \\
\hline 4 & $134(16,4)$ & $61(7,5)$ & $73(8,9)$ \\
\hline 5 & $77(9,4)$ & $43(11,9)$ & $34(7,5)$ \\
\hline \multicolumn{4}{|c|}{ Autopercepción del desempeño académico } \\
\hline Excelente & $39(4,8)$ & $21(2,6)$ & $18(2,2)$ \\
\hline Muy Bueno & $216(26,5)$ & $92(11,3)$ & $124(15,2)$ \\
\hline Bueno & $414(50,7)$ & $185(22,7)$ & $229(28,1)$ \\
\hline Satisfactorio & $109(13,4)$ & $45(5,5)$ & $64(7,8)$ \\
\hline No satisfactorio & $34(4,2)$ & $16(2)$ & $18(2)$ \\
\hline \multicolumn{4}{|c|}{ Autopercepción del estado de salud } \\
\hline Excelente & $125(15,3)$ & $89(10,9)$ & $36(4,4)$ \\
\hline Muy Bueno & $244(29,9)$ & $118(14,5)$ & $126(15,4)$ \\
\hline Bueno & $336(41,2)$ & $119(14,6)$ & $217(26,6)$ \\
\hline Regular & $96(11,8)$ & $26(3,2)$ & $70(8,6)$ \\
\hline Malo & $5(0,6)$ & $3(0,4)$ & $2(0,2)$ \\
\hline
\end{tabular}

Respecto al consumo de tabaco se encontró que 120 estudiantes $(15 \%)$ manifestaron que en la actualidad son fumadores, mientras que 696 estudiantes (85\%) manifestaron no fumar en la 
actualidad. Al preguntar acerca de la frecuencia de consumo de uno o más cigarrillos, en quienes manifestaron ser fumadores, 56 estudiantes respondieron que fuman una o dos veces al mes, 23 estudiantes lo hacen semanalmente, 27 casi diariamente; mientras que 14 fuman uno o más cigarrillos diariamente. Por su parte, 215 estudiantes (26\%) se describieron como bebedores frecuentes, frente a 601 estudiantes (74\%) que se consideraron como bebedores muy ocasionales (sólo en ocasiones especiales) o no bebedores. Al preguntar a los estudiantes que se describieron como bebedores frecuentes, por el número de días que ha bebido en las últimas dos semanas, 16 estudiantes respondieron no haberlo hecho, 114 estudiantes respondieron haberlo hecho entre uno y dos días, 67 estudiantes entre 3 y 5 días y 11 estudiantes manifestaron haber bebido entre 6 y 8 días durante las últimas dos semanas. La tabla 2 muestra la distribución de consumo de tabaco y alcohol a partir de algunos factores sociodemográficos.

Tabla 2. Asociación entre consumo de tabaco, alcohol y características sociodemográficas en estudiantes universitarios

\begin{tabular}{|c|c|c|c|c|c|c|c|c|}
\hline \multirow[t]{2}{*}{ Característica } & \multicolumn{2}{|c|}{ Fumador actual (n) } & \multirow[t]{2}{*}{$\mathbf{V}$} & \multirow[t]{2}{*}{$\mathbf{p}^{*}$} & \multicolumn{2}{|c|}{ Bebedor (n) } & \multirow[t]{2}{*}{ V Cramer } & \multirow[t]{2}{*}{$\mathbf{p}^{*}$} \\
\hline & $\mathbf{S i}$ & No & & & Frecuente & No & & \\
\hline \multicolumn{9}{|l|}{ Edad } \\
\hline 18 & 22 & 158 & & & 40 & 139 & & \\
\hline 19 a 21 & 54 & 290 & 0,04 & 0,722 & 93 & 250 & 0,04 & 0,851 \\
\hline 22 a 24 & 28 & 165 & & & 54 & 138 & & \\
\hline 25 a 50 & 16 & 83 & & & 27 & 72 & & \\
\hline \multicolumn{9}{|l|}{ Sexo } \\
\hline Femenino & 30 & 426 & & & 74 & 379 & & \\
\hline Masculino & 90 & 270 & 0,258 & 0 & 140 & 220 & 0,254 & 0 \\
\hline \multicolumn{9}{|l|}{ Año de estudio } \\
\hline 1 & 38 & 166 & & & 51 & 153 & & \\
\hline 2 & 28 & 205 & & & 58 & 175 & & \\
\hline 3 & 21 & 144 & 0,101 & 0,142 & 50 & 115 & 0,072 & 0,515 \\
\hline 4 & 16 & 118 & & & 32 & 102 & & \\
\hline 5 & 17 & 63 & & & 26 & 54 & & \\
\hline \multicolumn{9}{|l|}{ Facultad } \\
\hline Artes & 17 & 64 & & & 29 & 52 & & \\
\hline Agrarias & 11 & 14 & & & 9 & 16 & & \\
\hline Básicas & 3 & 33 & 0,195 & 0 & 12 & 24 & 0,138 & 0,018 \\
\hline Económicas & 1 & 42 & & & 8 & 35 & & \\
\hline Educación & 15 & 51 & & & 15 & 51 & & \\
\hline Ingenierías & 34 & 217 & & & 77 & 174 & & \\
\hline Salud & 39 & 275 & & & 66 & 248 & & \\
\hline \multicolumn{9}{|c|}{ Desempeño académico } \\
\hline Excelente & 7 & 32 & & & 12 & 27 & & \\
\hline Muy Bueno & 33 & 183 & & & 55 & 161 & & \\
\hline Bueno & 62 & 352 & 0,668 & 0,063 & 104 & 310 & 0,073 & 0,497 \\
\hline Satisfactorio & 16 & 93 & & & 35 & 74 & & \\
\hline No satisfactorio & 2 & 36 & & & 11 & 27 & & \\
\hline
\end{tabular}


Se destaca la asociación entre el sexo y el consumo de tabaco $(p<0,001)$ y consumo de alcohol $(p<0,001)$; siendo los estudiantes de sexo masculino los mayores consumidores. Además se relaciona la facultad y el tabaquismo $(\mathrm{p}<0,001)$ y el consumo de alcohol $(\mathrm{p}=0,018)$, encontrándose un mayor consumo, de manera proporcional al tamaño de la muestra, en los estudiantes de Ciencias Agrarias (44\% para el consumo de tabaco y $36 \%$ para el consumo de alcohol) y Artes (21\% y $36 \%$ respectivamente).

\section{Discusión}

Se ha establecido la prevalencia del consumo de tabaco y alcohol en un grupo de estudiantes universitarios, identificando asociación entre dichos hábitos y algunas características demográficas. Respecto al consumo de tabaco se encontró que un $15 \%$ de estudiantes son fumadores, frente a un $85 \%$ que no fuman. Este hallazgo es menor a los registrados en otros estudios realizados en estudiantes universitarios colombianos, como el de una universidad de Cartagena en el cual se reportó una prevalencia de consumo de $24 \%$, o el realizado por Cerezo et al.,15,16 en donde se determinó una prevalencia de consumo de cigarrillo de $22 \%$, o el trabajo realizado por García et al.,17 que registró un 17\% de consumo.

La prevalencia encontrada en el presente trabajo es mucho más baja respecto a otros estudios realizados en estudiantes universitarios peruanos $(\mathrm{p}=43 \%)$, chilenos (33\%), estadounidenses (35\%).13,18,9 Las cifras de los estudios realizados en Colombia referenciados en éste artículo son mayores que las registradas en el Estudio Nacional de Consumo de Sustancias Psicoactivas del año 2013. Según dicho estudio para el año 2013 se registró una prevalencia de consumo de tabaco de $16 \%$ en el grupo de personas entre $18 \mathrm{y}$ 34 años estimando que casi 1.800 .000 de jóvenes entre 18 y 34 años fuman en la actualidad. Los resultados de éste mismo estudio publicados en el año 2008 mostraban una prevalencia de consumo de tabaco de $21 \%$ para el grupo de edad entre los 18 y los 24 años y de 19\% para el grupo de edad entre 25 y 34 años.19,20
Aunque de manera leve, se aprecia, que comparando los resultados de los estudios colombianos hasta ahora referenciados con los del Estudio Nacional de Consumo de Sustancias Psicoactivas del año 2013 y los del presente trabajo, el consumo de tabaco en el grupo etario de interés ha disminuido, lo cual, podría estar relacionado con el éxito de las diferentes campañas antitabaquismo impulsadas en los últimos años a lo largo del país, que seguramente ha sensibilizado al grupo poblacional joven.

Sería importante indagar en las relaciones familiares de estos estudiantes para determinar si los hábitos desde el hogar también han tenido alguna incidencia en éstas cifras. Respecto a la frecuencia de consumo de uno o más cigarrillos, en quienes manifestaron ser fumadores, $47 \%$ de estudiantes respondieron que fuman una o dos veces al mes, $18 \%$ de estudiantes lo hacen semanalmente, $23 \%$ fuman casi diariamente; mientras que $12 \%$ fuman uno o más cigarrillos diariamente. Estas cifras son menores a las encontradas en otros estudios, en los que se ha registrado que el $30 \%$ o $40 \%$ de los estudiantes fumadores consumen más de dos cigarrillos al día. Aun así, es preciso recordar que dosis teóricamente pequeñas de cigarrillos consumidos al día también ejercen un impacto lesivo en la salud.13,9,21

Se ha determinado asociación entre el sexo y el consumo de tabaco, siendo el sexo masculino el mayor consumidor. Esta relación se ha encontrado en otros estudios.9,10 Sin embargo, para ambos sexos, el tabaquismo incide altamente en el síndrome coronario agudo y en la ocurrencia de la enfermedad obstructiva crónica.22,23

La prevalencia de consumo de alcohol encontrada en la muestra de estudiantes fue de $26 \%$, cifra relativamente baja en comparación con la reportada por otros trabajos, en los cuales las cifras de consumo de alcohol en estudiantes universitarios oscilan entre el 44 y el 58\%.8,13,18,24

Al igual que con el consumo de tabaco, al comparar con el Estudio Nacional de Consumo de Sustancias Psicoactivas del año 2013, nuestro 
resultado es alentador, ya que según dicho estudio, la mayor concentración de personas que consumen alcohol (aproximadamente 8'400.000) se encuentra entre los 18 y 34 años, de las cuales cinco millones son hombres, estableciendo en el grupo de 18 a 24 años, una prevalencia de $49 \%$ y en el grupo de 25 a 34 años de 45\%.19

Estos datos son mayores que los registrados por el mismo estudio en el año 2008 (46\% en el grupo de 18 a 24 años, $43 \%$ en el grupo de 25 a 34 años) lo cual enciende las alarmas por las consecuencias que se desencadenan del consumo excesivo de alcohol.20

En el presente trabajo se ha encontrado relación ente el sexo y el consumo de alcohol, con mayor prevalencia en los hombres, igual que en otros trabajos, en los que se ha registrado que en los países latinos muchas veces se alienta el consumo de alcohol entre varones, mientras que las mujeres se abstienen de ello.25,26 Otras investigaciones en estudiantes universitarios, han descrito, que en comparación con las mujeres, los hombres beben con mayor intensidad, experimentando de manera más frecuente sus consecuencias negativas y complicaciones. 27

Las consecuencias del consumo excesivo también difieren entre sexo. Los hombres tienden a experimentar conductas antisociales (discusiones, riñas, lesiones personales, accidentes, vandalismo), mientras que en las mujeres las consecuencias se traducen en el ámbito privado y personal (actividad sexual no consentida, pérdida de la memoria, autolesiones).28

Al preguntar a los estudiantes que se describieron como bebedores frecuentes, por el número de días que ha bebido en las últimas dos semanas, $8 \%$ de estudiantes respondieron no haberlo hecho, 55\% de estudiantes respondieron haberlo hecho entre uno y dos días, mientras que $37 \%$ de estudiantes manifestaron haber bebido entre $3 \mathrm{y}$ 8 días durante las últimas dos semanas, cifras similares a las reportadas en otros estudios.24

Aunque no se encontró relación entre el año de estudio y el consumo de tabaco o alcohol, son los estudiantes de primer y quinto año quienes reportan mayor consumo de éstas sustancias. A partir de los primeros años se registra un porcentaje de consumo, el cual se mantiene durante los siguientes años. Otros autores han encontrado que el consumo de alcohol disminuye a medida que incrementan los años de estudio, seguramente por la adaptabilidad al estrés que genera la vida académica y al conocimiento que se adquiere respecto a los efectos nocivos que lleva consigo el consumo de alcohol.11

Respecto al hábito de fumar, cuanto antes comienza una persona a fumar, mayor es el riesgo de convertirse en un fumador regular, desarrollar dependencia y sufrir consecuencias a largo plazo.12

Factores como la facilidad de acceso, la curiosidad, la presión de los amigos, la errada percepción de los jóvenes respecto a que fumar y beber alcohol representan autonomía y éxito, indicen en la adherencia precoz.12,29,30 Aunque el bajo desempeño académico también se ha descrito como un factor asociado en éste caso no resultó relación. ${ }_{13}$ En este estudio, además se ha encontrado asociación entre la facultad y el consumo de tabaco y alcohol.

Diversos investigadores han centrado su atención en la descripción de éstos hábitos en estudiantes universitarios del área de la salud.8,11,13,31 Justamente son ellos, los futuros profesionales quienes proporcionarán información acerca de los efectos de éstas sustancias, así como también soporte para su disminución y prevención de consumo. Aun así, fueron los estudiantes de las facultades de Ciencias Agrarias y Artes, los mayores consumidores de tabaco y alcohol.

A partir de las relaciones descritas anteriormente, se sugiere que las estrategias de intervención para el abordaje del tabaquismo y el consumo de alcohol dentro de la universidad incluyan una efectiva sensibilización sobre los riesgos de éstas conductas en la salud y la calidad de vida en ambos sexos, el fomento de los ambientes libres de humo, la protección a los no fumadores, la modificación de la aceptación social del acto de fumar y beber licor en exceso con lemas como 
"Universidad libre de alcohol y humo de tabaco" o "Mi facultad sin licor ni cigarrillo"; la creación de una red de identificación, orientación y tratamiento de los casos de abuso y dependencia detectados; la inclusión de temáticas relacionadas dentro de los planes académicos en los que se consideren hábitos, creencias y actitudes que faciliten hacia el futuro un soporte adecuado que les permita a los universitarios desempeñarse con suficiente criterio y convicción frente a esta problemática y la coordinación con la administración municipal para considerar la conveniencia de horarios de funcionamiento de bares y lugares de expendio de licor y tabaco que sean de alta concurrencia por parte de los universitarios cualquier día de la semana.

\section{Conclusiones}

Este estudio representa un aporte al diagnóstico de los estilos de vida de los estudiantes universitarios. Se encontró que la prevalencia de consumo de tabaco y alcohol se encuentra en un nivel bajo respecto a lo reportado en estudios previos. Aun así, estos hallazgos invitan a implementar políticas tendientes a potenciar a la Universidad como un entorno promotor de la salud de la comunidad educativa. Si bien estos resultados muestran que no existe un consumo problemático, se requiere promover estrategias en la comunidad educativa a través de programas efectivos en prevención del consumo de tabaco y alcohol focalizados y diferenciados por facultad y sexo, así como la identificación e intervención temprana en estudiantes con alto consumo. Esto permitiría ganar tiempo al desarrollo de futuras complicaciones generadas por factores que de una u otra forma podrían ser modificados, además de propiciar el desarrollo de adultos sanos capaces de revertir la tendencia ascendente que actualmente muestra el desarrollo de las enfermedades crónicas.

\section{Limitaciones}

Este fue un estudio descriptivo y por lo tanto no pueden establecerse inferencias causales a partir de los datos obtenidos. La información se ha obtenido a través de cuestionarios autodiligenciados, por lo que su validez podría estar condicionada de acuerdo a la actitud y honestidad de los sujetos que participaron en el estudio.

Conflicto de Intereses. Los autores declaran que no existen conflictos de intereses.

\section{Referencias}

1. Ansa V, Oyo-Ita A, Essien O. Perception of ischaemic heart disease, knowledge of and attitude to reduction of its risk factors. East Afr Med J. 2007;84(7):318-23.

2. Familoni IF, Familoni OB. Determinants and perception of cardiovascular risk factors among secondary school teachers in Oyo state Nigeria. East Afr Med J. 2011;40(4):339-43.

3. Dunbar A, Gotsis W, Frishman W. Second-hand tobacco smoke and cardiovascular disease risk: an epidemiological review. Cardiol Rev. 2013;2:94-100.

4. World Health Organization. Global status reporto in alcohol and health. Luxembourg, WHO; 2014.

5. Veloza M, Simich L, Strike C, Brands B, Giesbrecht N, Khenti A. Medio social y uso simultáneo de alcohol y tabaco en estudiantes universitarios de pregrado de carreras de ciencias de la salud de una universidad, Cundinamarca - Colombia. Texto Contexto Enferm, Florianópolis. 2012; 21(Esp):41-8.

6. Steptoe A, Wardle J. Health behaviour, risk awareness and emotional well-being in students from eastern Europe and western Europe. Soc Sci Med. 2001; 53:1621-1630.

7. Steptoe A, Wardle J, Cui W, Baban A, Glass K, Pelzer K. An international comparison of tobacco smoking, beliefs and risk awareness in university students from 23 countries. J Addict. 2002;97:1561-1571.

8. Mantilla SC, Gómez A, Hidalgo M. Actividad física, tabaquismo y consumo de alcohol en un grupo de estudiantes universitarios. Rev Salud Pública. 2011;13(5):748-58.

9. Berg C, Ling P, Hayes R, Berg E, Nollen N, Nehl E, Choi W, Ahluwalia J. Smoking frequency among current college student smokers: distinguishing characteristics and factors related to readiness to quit smoking. Health Educ Res. 2012;27(1):141-150.

10. Tirodimos I, Georgouvia I, Savvala TN, Karanika E, Noukari D. Healthy lifestyle habits among Greek university students: differences by sex and faculty of study. East Mediterr Health J 2009;15(3):722-728.

11. Valle R, Sánchez E, Perales A. Sintomatología depresiva y problemas relacionados al consumo de alcohol durante la formación académica de estudiantes de medicina. Rev Peru Med Exp Salud Pública. 2013;30(1):54-7.

12. Navarro M, Espig H, Medina V. Consumo de tabaco en estudiantes universitarios de nuevo ingreso a escuelas de Ciencias de la Salud. Rev Salud Pública. 2010;14(1):51-60. 
13. Lorenzo M, Cajaleón B, Gutiérrez E. Prevalencia y factores asociados al consumo de alcohol y tabaco en estudiantes de nutrición de una universidad de LimaPerú. Revista Peruana de Epidemiología. 2012;16(3):15.

14. Centers for Disease Control and prevention. The global school and health survey backround. 2013.

15. Hernández J, Herazo Y, Valero M. Frecuencia de factores de riesgo asociados a enfermedades cardiovasculares en población universitaria joven. Rev. Salud Pública. 2012;12(5):852-864.

16. Cerezo M, Vergara M, Nieto E, Cifuentes O, Parra J. Características de salud pública de una universidad privada de la ciudad de Manizales. Rev. Promoción Salud. 2011;16(1):73-86.

17. García M, García J. Cardiovascular risk factor prevalence in university students Rev. Salud Pública. 2012;14(5):822-830.

18. Morales G, Del Valle C, Belmar C, Orellana Y, Soto A, Ivanovic D. Prevalencia de consumo de drogas en estudiantes universitarios que cursan primer y cuarto año. Rev. Med Chile. 2011;139:1573-1580.

19. Ministerio de Justicia y del Derecho. Observatorio de drogas de Colombia. Ministerio de Salud y Protección Social. Estudio Nacional de consumo de sustancias psicoactivas en Colombia. 2013.

20. Ministerio de la Protección Social. Dirección Nacional de Estupefacientes. Estudio Nacional de consumo de sustancias psicoactivas en Colombia. 2008.

21. Anchique CV, Aristizábal D, Aschner P, Jimena D, Castillo VR, Castro JM, et al. Guías de prevención primaria en riesgo cardiovascular. Tópicos selectos. Rev. Col. de Card. 2009;16(Sup.3):71-196.

22. Gorbatchev D, Ramírez A, Maya M, Sansores R, Guzmán A, Regalado J. Prevalencia de dislipidemia en los fumadores que acuden a un programa de ayuda para dejar de fumar. Revista del Instituto Nacional de Enfermedades Respiratorias. 2005;19(2):102-107.

23. Nazir SA, Erbland ML. Chronic obstructive pulmonary disease: an update on diagnosis and management issues in older adults. Drugs Aging. 2009;26(10):81331.

24. Zakletskaia L, Wilson E, Francis M. Alcohol Use in Students Seeking Primary Care Treatment at University Health Services. Am Coll Health. 2010;59(3):217-223.

25. Wagner G, Stempliuk V, Zilberman M, Barroso L, Andrade A. Alcohol and drug use among university students: gender differences. Rev Bras Psiquiatr. 2007;29:123-9.

26. Chau C, Oblitas H. Características del consumo de bebidas alcohólicas en estudiantes universitarios. Revista peruana de drogodependencias. 2007;5:43-70.

27. Escobar M, Maín M. Consumo de alcohol y tabaco en estudiantes de pregrado de una universidad privada de Medellín, 2007. Invest Educ Enferm. 2009;27(1):60-68.

28. Diaz A, Diaz R, Hernández C, Narro J, Fernández H, Solís C. Prevalencia del consumo riesgoso y dañino de alcohol y factores de riesgo en estudiantes universitarios de primer ingreso. Rev. Salud Ment. 2008;31:271-282.
29. Araneda J, Cumsille F. Consumo de drogas en población escolar de Chile, en alumnos de 8 básico a 4 medio. Rev. Chil Salud Pública. 2004;8(2):63-71.

30. Arillo-Santillan E, Fernández E, Hernández M,Tapia M, Cruz A, Lazcano E. Prevalencia de tabaquismo y bajo desempeño escolar en estudiantes de 11 a 24 años de edad del estado de Morelos, México. Revista Salud Pública Mex. 2002; 44(supl)1:S54-S66.

31. Almutairi K. Prevalence of tobacco use and exposure to environmental tobacco smoke among saudi medical students in riyadh, Saudi Arabia. J Community Health. 2014;39:668-673. 